\title{
Microscale Thermophoresis in Liquids Induced by Plasmonic Heating and Characterized by Phase and Fluorescence Microscopies
}

\author{
SUPPLEMENTARY INFORMATION
}

\author{
Sadman Shakib, ${ }^{1}$ Benoit Rogez, ${ }^{1}$ Samira Khadir, ${ }^{1}$ Julien Polleux, ${ }^{2}$ Alois Würger, ${ }^{3}$ and Guillaume Baffou ${ }^{1, *}$ \\ ${ }^{1}$ Institut Fresnel, CNRS, Aix Marseille University, Centrale Marseille, Marseille, France \\ ${ }^{2}$ Department of Ophthalmic Optics, FH Gesundheit, \\ Health University of Applied Sciences Tyrol, 6020 Innsbruck, Austria \\ ${ }^{3}$ Laboratoire Ondes et Matière dAquitaine, Université de Bordeaux 6 CNRS, 33405 Talence, France
}

\section{A. Fabrication of gold nanoparticle samples}

We have used gold nanoparticle samples made by block copolymer micellar lithography (BCML). This bottomup chemical synthesis technique is described in detail in Ref. [1]. Briefly, micelles composed of a gold ion core and an organic capsule are synthesized by mixing a copolymer with $\mathrm{HAuCl}_{4}$ in toluene. Then, the micelles are deposited on a cleaned glass coverlips by dip-coating in toluene. The deposited micelles are then reduced by UV illumination in the presence of a reducing agent to produce gold seeds. Finally, gold seeds are grown by putting the coverslip in contact with an aqueous solution of $\mathrm{KAuCl}_{4}$ and ethanolamine for $16 \mathrm{~min}$, leading to a quasiperiodic and

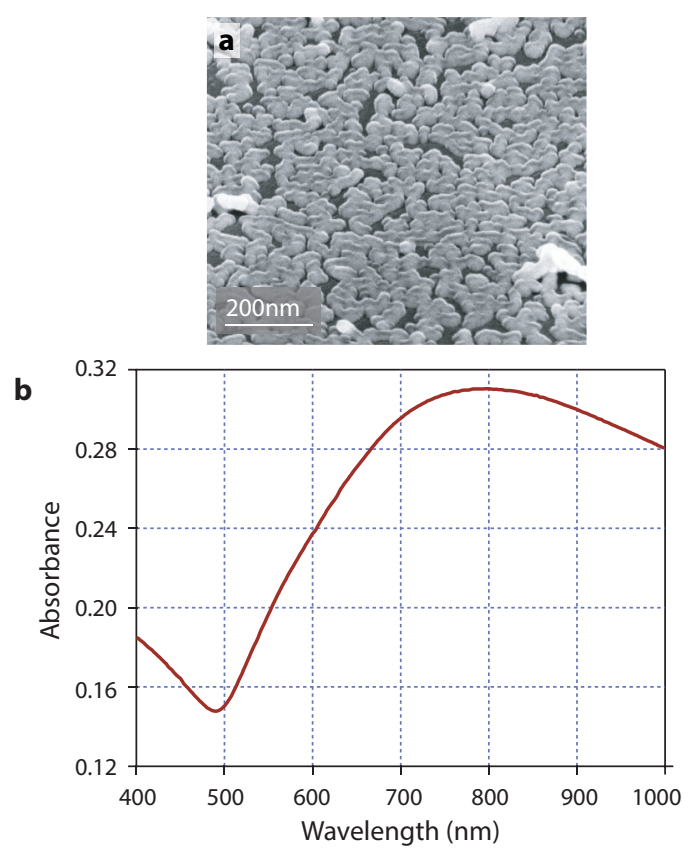

FIG. S1: (a) SEM image (with a $45^{\circ}$ tilt angle) of brain shaped structure of gold nanoparticles prepared by BCML method. (b) Absorption spectra of the nanaoparticle sample.

\footnotetext{
*Electronic address: guillaume.baffou@fresnel.fr
}

very uniform arrangement of nonspherical gold nanoparticles absorbing in the near-infrared range. The shapes are enlarged potatoes, almost merged creating a brainlike structure (Fig. S1).

From this data set, the inverse function $T=\gamma(n)$ can be determined by interpolation, or by using a fitting polynomial, from which the temperature map can be calculated using the expression

$$
T(x, y)=\gamma(n(x, y))=\gamma\left(\delta \ell(x, y) / h+n_{0}\right)
$$

\section{B. Photo-, thermo-bleaching and temperature dependence of the fluorescent particles.}

We used fluorescence intensity to render variations of particle concentration, but fluorescence can vary for other reasons, namely photobleaching, thermobleaching and temperature variation.

Figure S2a quantifies photobleaching effects. Since photobleaching may depend on temperature, we varied the sample temperature using the VAHEAT system from Interherence GmbH. The VAHEAT substrate is endowed with a resistive part and a thermocouple that enables the precise temperature control of the liquid, up to $105^{\circ} \mathrm{C}$, by resistive heating of the substrate. In this experiment, a $44-\mu \mathrm{m}$ thick liquid layer containing the fluorescent nanobeads at a concentration of $88 \mathrm{nM}$ was continuously illuminated with a 470-nm light with an irradiance of $4.4 \times 10^{-4} \mu \mathrm{W} / \mu \mathrm{m}^{2}$. Fluorescence intensity was monitored using video imaging of the sample plane, and the heating was successively turned off and on during $10 \mathrm{~min}$ (Fig. S2a). The photobleaching rates was estimated to be around $0.0055 \% / \mathrm{s}$ at $21^{\circ} \mathrm{C}$, and $0.011 \% / \mathrm{s}$ upon heating at $40^{\circ} \mathrm{C}$. These rates were negligible in our study, as expected for fluorescent beads.

To investigate in detail the question of pure thermobleaching, we had to cancel photobleaching. For this purpose, we did not excite fluorescence using cw illumination. We sent 2 -s long flashes of light only during fluorescence measurement to avoid photobleaching between the measurements. In Fig. S2b, we can see the onset of heating reduces the fluorescence intensity by very little and then doesn't decrease the counts while heating as in Fig. S2a. 

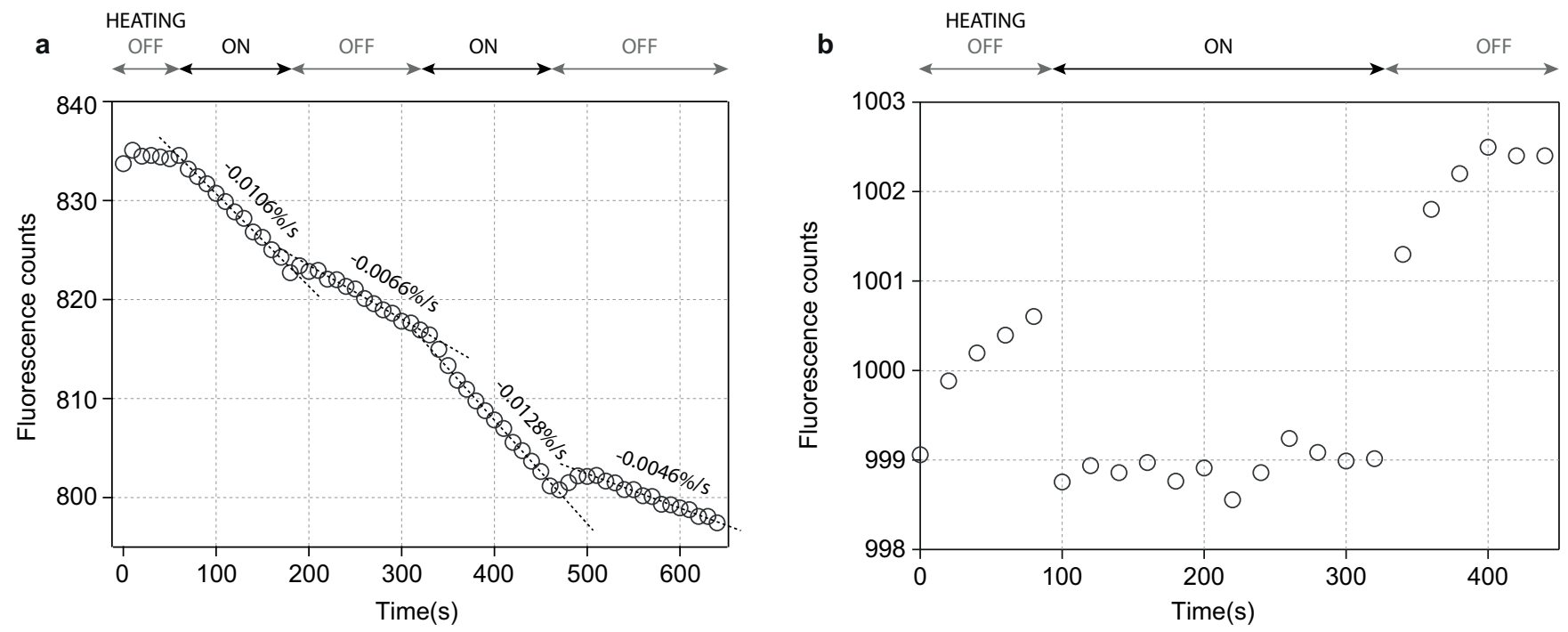

FIG. S2: (a) Photobleaching of a nanobead solution under continuous illumination at room temperature. Fluorescence decay follows a linear trend, which is accelerated upon heating at $40^{\circ} \mathrm{C}$. (b) Variation of the fluorescence intensity over time, showing a decay of fluorescence brightness upon heating at $30^{\circ} \mathrm{C}$.

From this study we can draw the conclusion that thermobleaching does not occur. Temperature increases just favor photobleaching. However, the fluorescence brightness decreases by $0.2 \%$ when the temperature rises by $10^{\circ} \mathrm{C}$. Note that above $T=40^{\circ} \mathrm{C}$, microspheres seem to undergo some irreversible conformational changes. So we decided to conduct our experiments below $40^{\circ} \mathrm{C}$.

\section{Temperature retrieval algorithm}

The map of optical path difference $\delta \ell$ measured by QLSI depends on the refractive index distribution following

$$
\delta \ell(x, y)=h\left[n(x, y)-n_{0}\right]
$$

where $n_{\infty}$ is the refractive index of the liquid at ambient temperature $T_{\infty}$.

The refractive index of the medium due to temperature variation can thus be directly mapped using

$$
n(x, y)=\delta \ell(x, y) / h+n_{0}
$$

Datasets of the temperature dependence of the refractive indices of liquids can be found in the literature. For water, we used data from Schiebener et al.[2], at $\lambda=632$ $\mathrm{nm}$, represented in Fig. S3a.

[1] F. Kundrat, G. Baffou, and J. Polleux, Nanoscale 7, 15814

Ref. Data 14, 933 (1985). (2015).

[2] I. Thormählen, J. Straub, and U. Grigull, J. Phys. Chem. 

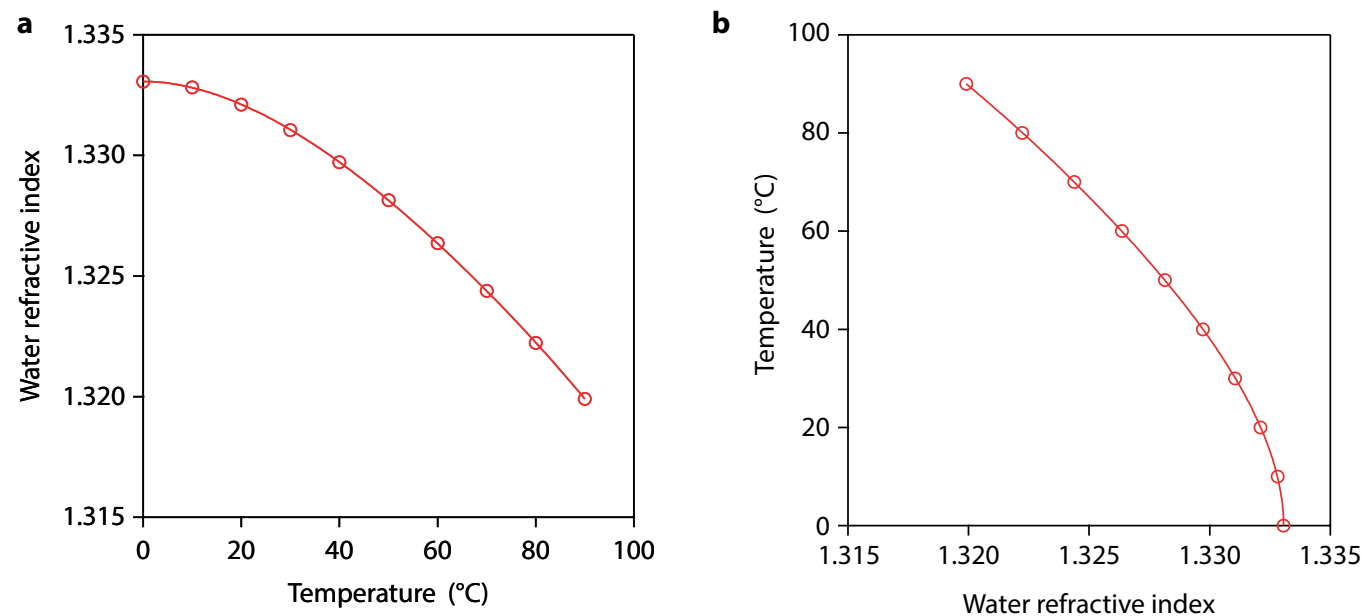

FIG. S3: (a) Refractive index of water as a function of the temperature for a wavelength of $\lambda=632 \mathrm{~nm}$, at ambient pressure Data taken from Ref. [2] (b) Inverse of the previous graph, which plots the temperature as a function of the refractive index. This line shape is used to convert the measure refractive index map into a temperature map. 